\title{
La transformación de la seguridad en Europa desde la caída del muro de Berlín. Una revisión crítica
}

\author{
The Transformation of Security in Europe since \\ the fall of the Berlin Wall. A Critical Review
}

\author{
Alberto Priego \\ U.P. Comillas \\ apriego@comillas.edu
}

Sumario: I. Introducción.-II. Estado de la Cuestión.-III. El Análisis de la Transformación Europea tras la caída del Muro de Berlín. 1. Adaptación a la nueva situación (1989-2001). 1.1. La importancia de los antiguos aliados soviéticos. 1.2. La percepción de la arquitectura de seguridad. 1.3. Los conflictos en Europa. 2. La Guerra al Terrorismo (2001-2004). 2.1. La importancia de los antiguos aliados soviéticos. 2.2. La percepción de la arquitectura de seguridad. 2.3. Los conflictos en Europa. 3. Final de la Luna de Miel (2005-2013). 3.1. Adaptación a la nueva situación. 3.1. La importancia de los antiguos aliados soviéticos. 4. Divorcio y comienzo de la escalada (2014-2019). 4.1. La importancia de los antiguos aliados soviéticos. 4.2. La percepción de la arquitectura de seguridad. 4.3. Los conflictos en Europa.IV. Conclusiones. - V. Bibliografía

Resumen: En el año 1989 el símbolo de la división de Europa desaparecía gracias a un conjunto variado de circunstancias que convirtieron esa situación en insostenible. Uno de los ámbitos que más se vieron afectados fue el de la seguridad. Hoy 30 años después Europa sigue dividida en dos bloques y el nivel de enfrentamiento entre Moscú y Bruselas ha alcanzado cotas inimaginables a pesar de o quizás porque la OTAN se ha ampliado hasta la misma frontera con Rusia. Este trabajo pretende hacer una revisión de la literatura sobre seguridad europea al tiempo que dar una perspectiva coherente de lo que ha ocurrido en estos 30 años en los que Europa vive sin el Muro de Berlín. Para ello se dividirá el periodo elegido en cuatro períodos (1989-2001, 2001-2004, 2005-2013 y 2013 en adelante) en los que se estudiarán los siguientes aspectos: la importancia de los antiguos aliados soviéticos, la percepción de la arquitectura de seguridad y los conflictos en Europa.

Palabras clave: Muro de Berlín, Rusia, OTAN, UE y OSCE 
Abstract: In the year 1989 the symbol of the division of Europe disappeared thanks to a varied set of circumstances that turned this situation into untenable. One of the most affected areas was security. Today, 30 years later, Europe remains divided into two blocs and the level of confrontation between Moscow and Brussels has reached unimaginable levels despite or perhaps because of NATO expansion up to the border of Russia. This work aims to make a review of the literature on European security while giving a coherent perspective of what has happened in these 30 years in which Europe has lived without the Berlin Wall. To this end, the chosen period will be divided into four periods (1989-2001, 2001-2004, 2005-2013 and 2013 onwards) in which the following aspects will be studied: the importance of the former Soviet allies, the perception of the security architecture and conflicts in Europe.

Keywords Berlin Wall, Russia, NATO, EU and OSCE.

\section{Introducción}

El 9 de noviembre de 1989 los ciudadanos de Berlín Oriental asaltaban el muro que durante décadas les había separado de sus hermanos de la zona Occidental. Detrás quedaban las historias de los 3000 alemanes que fueron detenidos intentado saltar al otro lado y sobre todo, las ilusiones de las 200 personas que perdieron su vida intentando tener una mejor en la Alemania Occidental. En total, se calcula que 3.5 millones de alemanes huyeron a la RFA en los más de 50 años que permanecieron separados, lo que supuso que la RDA perdió un $20 \%$ del total de su población.

Más allá del hecho de la caída del Muro de Berlín, el nueve de noviembre de 1989 pasó a la historia como el día en el que se inició la transformación de la seguridad en Europa. Tras el discurso de Gorbachov en la Asamblea General de la ONU (1986) que ponía fin a la Doctrina Breznev, los estados satélites de la URSS comenzaron su carrera para integrarse en las estructuras de seguridad occidental: OTAN y UE. Esta redistribución de las capacidades entre los dos grandes polos que se establecieron tras la Segunda Guerra Mundial, provocó en un primer momento un gran desconcierto que poco a poco fue diluyéndose para adaptarse a una nueva realidad en la que ya no existía ni la URSS, ni el Pacto de Varsovia y donde buena parte de los PECOs ${ }^{1}$ se integraron en las estructuras de segu-

${ }^{1}$ Se decidió utilizar el concepto Países de Europa Central y Oriental (PECOs) para evitar la denominación Europa del Este ya que además de no ser correcta desde el punto de vista geográfico planteaba una connotación política. 
ridad Euro-Atlánticas. Debido a la magnitud de la transformación que sufrió Europa a finales del siglo pasado, la producción bibliográfica ha sido muy abundante y en la medida de lo posible vamos a tratar de hacer una revisión de ésta, asumiendo de antemano que resultaría imposible hacer un repaso completo de todos los trabajos publicados.

\section{Estado de la Cuestión}

Debido a la gran relevancia que tuvo el tema para Europa, la producción de literatura científica fue inmensa por lo que antes de iniciar su revisión es necesario advertir, de nuevo, que ésta no será completa. Por este motivo, se ha decidido utilizar dos criterios para priorizar la revisión de los trabajos. En primer lugar, los trabajos que aborden el estudio de la cuestión desde la Historia, desde las Relaciones Internacionales y en menor medida desde la Ciencia Política. En segundo lugar, se atenderán prioritariamente aquellos trabajos publicados en España y en menor medida en el Reino Unido y Estados Unidos. De este modo, aunque soy consciente de que la labor seguirá siendo titánica, al menos se habrá reducido en algún grado su inmensidad.

En los primeros momentos tras la caída del Muro de Berlín - e incluso antes - ya comenzaron a publicarse una serie de trabajos que trataban de explicar los problemas de la URSS o - posteriormente- las causas de su extinción. Algunos autores como Hewett ${ }^{2}$, Goldman ${ }^{3}$ o Rutland ${ }^{4}$ centraron sus investigaciones en los problemas económicos, otros como Colins ${ }^{5}$, Morewood $^{6} \mathrm{o}$ Watson ${ }^{7}$ en la figura de Gorbachov y otros muchos como Taibo ${ }^{8}$ y Odon $^{9}$ entendieron que fue precisamente la cuestión de la seguridad la que llevó a la URSS a la extinción. Tampoco faltaron autores como Ricardo

${ }^{2}$ Edward A, Hewett, Reforming the Soviet Economy: Equality Versus Efficiency (Washington D.C: Brookings Institution, 1990).

${ }^{3}$ Marshall Goldman, What Went Wrong With Perestroika, (New York: W. W. Norton, 1991).

${ }^{4}$ Robert Rutland, The Myth of the Plan: Lessons of Soviet Planning Experience, (London: Hutchinson, 1985)

5 Alan R. Collins, «Gorbachev and the End of the Cold War», Review of International Studies, no. 24 (1998).

6 Steven Morewood, «Gorbachev and the Collapse of Communism», History Review, no. 31 (1998): 201-219.

7 William Watson, The Collapse of Communism in the Soviet Union, (Westport: Greenwood, 1998).

${ }^{8}$ Carlos Taibo, La Unión Soviética de Gorbachov, (Madrid: Fundamentos, 1989).

9 William Odom, The Collapse of the Soviet Military, (New Haven: Yale University Press, 2000). 
Martín de la Guardia y Guillermo Pérez ${ }^{10}$ que buscaron una aproximación más holística para explicar el final del imperio Soviético. Probablemente utilizando el famoso título de Jaques Levesque ${ }^{11}$, las verdaderas causas que llevaron a la URSS a su fin son todavía un enigma. En los últimos años hemos asistido a una nueva ola de publicaciones que, tratan de aportar nuevas perspectivas sobre los últimos años de la URSS. Entre otros muchos podemos destacar a Pravda ${ }^{12}$, Sunny ${ }^{13}$, Plokhy ${ }^{14}$ o Edele ${ }^{15}$ como ejemplo de este nuevo interés por los estudios soviéticos.

Una vez superado el shock que supuso el derrumbe de la URSS, muchos autores como Esther Barbé ${ }^{16}$ o Martín De La Guardia ${ }^{17}$ comenzaron a preguntarse por la configuración de ese nuevo orden mundial que parecía superar la bipolaridad. Sin embargo, con el paso de los años han ido surgiendo autores como Lukas ${ }^{18}$ que siguen defendiendo que aun nos encontramos ante una nueva Guerra Fría u incluso otros ${ }^{19}$ que afirman que todavía no habríamos superado la misma. Estos dos argumentos que en buena medida son uno, serían la base del actual enfrentamiento entre la Federación Rusa y la OTAN.

Precisamente la OTAN ha sido uno de los temas que ha centrado un mayor número publicaciones. Son muchos los autores que han trabajado en el estudio de la Alianza Atlántica destacando a Yost ${ }^{20} \mathrm{o}$ Asmus $^{21}$. Entre los

10 Ricardo Martín de la Guardia y Guillermo Pérez: La Unión Soviética de la perestroika a la desintegración, (Madrid: Edición Istmo, 1996).

11 Jacques Levesque, The Enigma of 1989: The USSR and the Liberation of Eastern Europe, (Berkley: University of California Press, 1997).

12 Alex Pravda, «The collapse of the Soviet Union, 1990-1991» en Melvyn Leffler y Odd Arne Westad: The Cambridge History of the Cold War, (Cambridge: Cambridge University Press, 2010): 356-377.

13 Ronald G. Sunny, The Soviet Experiment: Russia, the USSR, and the Successor States. (Oxford: Oxford University Press, 2010).

${ }^{14}$ Serhii Plokhy, The Last Empire: The Final Days of the Soviet Union, (London: Basic Book, 2015).

15 Mark Edele, The Soviet Union: A Short History,(London: Willey, 2016).

${ }^{16}$ Esther Barbé: La seguridad en la nueva Europa, (Madrid: La Catarata, 1996).

17 Ricardo Martín de la Guardia, «Consideraciones sobre el nuevo orden mundial después de 1989», Historia Abierta, 26 (2000): 2-8; Ricardo Martín de la Guardia, 1989, el año que cambió el mundo los orígenes del orden internacional después de la Guerra Fría, (Tres Cantos: AKAL, 2012).

${ }^{18}$ Edward Lukas The New Cold War: Putin's Russia and the Threat to the West, (London: St Martin Griffin, 2014).

19 Robert Legvold, The Return to the Cold War, (London: Polity, 2016).

20 David S. Yost, «The new NATO and collective security», Survival, no. 2 (1998): 13560. David S. Yost, NATO Transformed: The Alliance's New Roles in International Security, (Washington D.C: United States Institute of Peace, 1999)

${ }^{21}$ Ronald D. Asmus, Opening NATO's Door. How the Alliance Remade Itself for a New Era, (New York: Columbia University Press, 2002). 
temas tratados destaca sobre todo el impacto de las ampliaciones tanto en la propia organización como en las relaciones de ésta con Rusia ${ }^{22}$. En España Antonio Marquina ${ }^{23}$ ha sido el autor que mayor atención le ha prestado a la organización, aunque también deben ser destacado otros como Delage ${ }^{24}$, Caracuel $^{25}$, Arteaga ${ }^{26}$ o Corchado y Sanz ${ }^{27}$. También debemos mencionar a algunos autores españoles como Taibo ${ }^{28}$ o Hernandez Holgado ${ }^{29}$ que sin dejar de estudiar la transformación de la organización, han planteado una visión crítica con la OTAN, sobre todo tras la intervención en Kosovo.

$\mathrm{Si}$ bien los trabajos de estos autores analizan los cambios en la organización tras la caída del Muro de Berlín, éstos parecen minúsculos si los comparamos con las transformaciones que la Alianza tuvo que asumir con el 11-S. Por ello, se hizo necesario revisar los postulados planteados por esa primera generación de estudiosos y en ese sentido tanto autores clásicos como Sloan ${ }^{30}$, Hunter ${ }^{31}$ o Yost $^{32}$ como otros nuevos como Cross $^{33}$, Pothier ${ }^{34}$ o Frühling \& Lasconjarias ${ }^{35}$ comenzaron a trabajar en

22 Stephen Blank, «Russia, NATO Enlargement and Baltic States», World Affairs, no. 3 (1998):115-125; James Goldgeier, Not Whether but When: The U.S. Decision to Enlarge NATO. (Washington, DC: Brookings Institution Press, 1999); Thomas Szayna, NATO Enlargement, 2000-2015. Determinants and Implications for Defense Planning, (Santa Monica: RAND. 2001).

${ }^{23}$ Antonio Marquina y Tomás Mestre: «España y la OTAN», Historia no.16 (1985); Antonio Marquina (Ed.): El Flanco Sur de la OTAN, (Madrid: Ed. Complutense, 1992); Antonio Marquina, España en la estructura militar de la OTAN, (Madrid: UNISCI, 1998); Antonio Marquina, La OTAN en su medio siglo, (Madrid: ATA, 1999).

${ }^{24}$ Fernando Delage, «Del Tratado de Washington a la guerra de Kosovo: la OTAN cumple cincuenta años», Cuadernos de Historia Contemporánea, no. 21 (1999): 341-353.

${ }_{25}$ María A. Caracuel, Los cambios de la OTAN tras el fin de la Guerra Fría, (Madrid: Tecnos, 1997).

${ }^{26}$ Felix Arteaga, La seguridad de Europa occidental: 1986-1991. (Madrid: Biblioteca Nueva, 2001).

27 Manuel Corchado y Carlos Sanz, «La Alianza Atlántica: cincuenta años de visión desde España», Cuadernos de Historia Contemporánea, no. 22 (2000): 387-397.

${ }_{28}$ Carlos, Taibo, Para entender el conflicto de Kosova, Madrid, La Catarata, 1999.

${ }_{29}$ Fernando Hernández Holgado, Historia de la OTAN. De la guerra fría al intervencionismo humanitario, (Madrid: La Catarata, 2000).

${ }^{30}$ Stanley Sloan, Defense of the West: NATO, the European Union and the Transatlantic Bargain, (Manchester: Manchester University Press, 2016)

31 Robert Hunter, Sergey Rogov y Olga Oliker, NATO and Russia. Bridge-Building for the 21st Century Report of the Working Group on NATO-Russia Relations, (Santa Monica: RAND, 2001).

32 David S. Yost, NATO's Balancing Act, (Washington: United States Institute of Peace, 2014).

33 Sharyl Cross, «NATO-Russia Security Challenges in the Aftermath of Ukraine Conflict: Managing Black Sea Security and Beyond», Southeast European and Black Sea Studies, no. 2 (2015): 151-177.

${ }^{34}$ Fabrice Pothier, «An Area-Access Strategy for NATO», Survival, no. 3 (2017): 73-80.

35 Stephan Frühling and Guillaume Lasconjarias, «NATO A2/AD and the Kaliningrad Challenge», no. 2 (2016): 95-116. 
asuntos tales como las siempre complicadas relaciones con Rusia, el escudo antimisiles, la repuesta $\mathrm{A} 2 / \mathrm{AD}$ o las renacidas relaciones con la UE tras la Cumbre de Newport.

La evolución de Rusia como actor internacional también ha acaparado un buen número de publicaciones. Aunque en los primeros años muchos autores como Olga Oliker ${ }^{36}$, Dawisha o Parrot ${ }^{37}$ buscaron las causas del declive ruso, pronto comenzaron a surgir trabajos que trataban de explicar la nueva política exterior de Rusia, con especial interés a sus relaciones con OTAN y con los Estados que componían el «Extranjero Próximo ${ }^{38}$ ». Sin embargo, la llegada al poder de Putin supuso una verdadera transformación en la proyección exterior de Rusia lo que provocó un incremento exponencial del número de trabajos tanto centrados en el líder ruso ${ }^{39}$ como en los distintos aspectos en los que éste ha centrado su política exterior. Así podemos destacar las obras sobre los conflictos con Georgia ${ }^{40}$ con Ucrania ${ }^{41}$ y sobre todo las tensas relaciones con la OTAN ${ }^{42}$.

En lo que a la política de seguridad de la Unión Europea se refiere, también debemos destacar el gran interés que este tema ha tenido en la academia. Aunque, por motivos de espacio resulta imposible llevar a cabo una revisión completa de la literatura, podemos citar a $\mathrm{Hill}^{43}, \mathrm{Smith}^{44}$, Keukeleire ${ }^{45}$,

36 Olga Oliker, Assessing Russia's decline, (Santa Mónica: RAND, 2002).

${ }^{37}$ Karen Dawisha y Bruce Parrot, Conflict, Cleavage and change in Central Asia and the Caucasus, (Cambridge: Cambridge University Press, 1997)

38 Ver Dov Lynch, Russian peacekeeping in the CIS. The cases of Moldova, Georgia and Tajikistan, (London: MacMillan Press, 2000); Leon Aron y Kenneth M. Jensen, The emergence of Russian Foreign Policy, (Washington D.C: United States Institute of Peace Press, 1994); Roy Allison y Christoph Bluth, Security Dilemmas in Russia and Eurasia, (London: RIIA, 1998).

${ }^{39}$ Richard Sakwa, Putin: Russia's Choice, (London: Routledge, 2007); Mark Galeoti, We Need to Talk About Putin: Why the West gets him wrong, and how to get him right, (London:Ebury Book, 2019)

${ }^{40}$ Svante Cornell y Frederick Starr, The Guns of August 2008: Russia's War in Georgia -Studies of Central Asia and the Caucasus-, (Abingdon: Routledge, 2009).

${ }^{41}$ Sharyl Cross, «NATO-Russia Security Challenges in the Aftermath of Ukraine Conflict: Managing Black Sea Security and Beyond», Southeast European and Black Sea Studies, no. 2 (2015): 151-177.

${ }^{42}$ Robert Hunter, Sergey Rogov y Olga Oliker, NATO and Russia. Bridge-Building for the 21st Century Report of the Working Group on NATO-Russia Relations, (Santa Monica: RAND, 2001)

${ }^{43}$ Christopher Hill, «Renationalising or Regrouping: EU Foreign Policy since 11 September 2001», Journal of Common Market Studies, no.1 (2004): 143-163.

${ }^{44}$ Michael E. Smith, Europe's Common Security and Defence Policy, (Cambridge: Cambridge University Press, 2017) Michael E. Smith, Europe's Foreign and Security Policy: The Institutionalization of Cooperation, (Cambridge: Cambridge University Press, 2004).

45 Stephan Keukeleire, «Directorates in the CFSP/CESDP of the European Union: A Plea for a Restricted Crisis-Management Group», European Foreign Affairs Review, no.1 (2001). 
Merlinger ${ }^{46}$ o a Mérand ${ }^{47}$ como algunos de los autores más destacados en este campo. En España hay que mencionar - entre otros - muchos autores a Francisco Aldecoa ${ }^{48}$, a Barbé ${ }^{49}$ o a Natividad Fernández ${ }^{50}$. Desde una perspectiva histórica debemos resaltar a Pérez Bustamante ${ }^{51}$, a Gil Pecharromán ${ }^{52}$ o de nuevo a R. Martín de la Guardia y a Guillermo Pérez ${ }^{53}$. Todos estos trabajos si bien no son obras centradas en materia de seguridad y defensa, al hacer una revisión de todo el proceso de integración también abordan estas cuestiones.

La OSCE es quizás la organización de seguridad en Europa que menos interés ha suscitado entre los académicos. Entre todos los demás debemos subrayar la obra de Freire ${ }^{54}$ o las aportaciones de Galbreath ${ }^{55}$, Lenz $^{56}$ o Muller ${ }^{57}$.

Tal y como hemos visto, la literatura sobre seguridad europea es muy extensa. Sin embargo, quizás se echa de menos trabajos que revisen de forma conjunta la incidencia de la transformación en la seguridad que ha sufrido Europa tras la caída del Muro de Berlín. Es cierto que encontramos bastante trabajos centrados en la UE, muchos en la OTAN y algunos, aunque pocos en la OSCE. Sin embargo, no encontramos obras de carácter general que analicen

46 Michael Merlinger, EU Security Policy: What It Is, How It Works, Why It Matters, (London: Lynne Rienner Publishers, 2011).

47 Frédéric Mérand, European Defence Policy. Beyond the Nation State, (Oxford: Oxford University Press, 2008).

48 Francisco Aldecoa, «La política común de seguridad y defensa en la Constitución Europea: hacer creíble la política de responsabilidad», Cuadernos Europeos de Deusto, no. 34 (2006): 11-40; Francisco Aldecoa, «La Política Exterior y la Política Europea de Seguridad y Defensa en el Tratado de reforma: la consolidación de Política Exterior Común», Revista de las Cortes Generales, no. 70-72 (2007): 237-268.

49 Esther Barbé, Política exterior europea, (Barcelona: Ariel, 2000).

${ }^{50}$ Natividad Fernández, «La política exterior y la proyección externa de la Unión Europea. Impacto de los trabajos de la Convención Europea, Revista de Estudios Políticos, 119 (2003), pp. 381-416.

51 Rogelio Pérez Bustamante, Un siglo en la historia de la Unión Europea. 1914-2014: Cronología (Plural), (Valencia: Tirant Lo Blanc, 2014) Rogelio Pérez Bustamante, Historia Jurídica y Política de la UE, (Madrid: Edisofer, 2008).

52 Julio Gil Pecharromán, Historia de la Integración Europea, (Madrid: UNED, 2016).

53 Ricardo Martín de la Guardia y Guillermo Pérez, La Europa del Este. Del Telón de Acero a la integración en la Unión Europea, (Madrid: Biblioteca Nueva, 2002).

${ }^{54}$ María R. Freire, Conflict and security in the former Soviet Union: the role of the OSCE, (Abingdon: Routledge, 2003).

55 David Galbreath, The Organization for Security and Co-operation in Europe, (Abingdon: Routledge, 2007).

${ }^{56}$ Katrin Lenz, The role of the Organization for Security and Cooperation in Europe (OSCE) in today's European security architecture, GRIN Verlag, 2005.

57 Otto Muller, The Ukraine Crisis and Russia's Attitude towards the Organization for Security and Co-operation in Europa, GRIN Verlag, 2017. 
la transformación global de la seguridad en Europa, atendiendo sobre todo a la inclusión de los antiguos miembros del Pacto de Varsovia en las arquitecturas de seguridad europea. Una de los fenómenos que también ha cambiado el concepto de seguridad en Europa ha sido la vuelta de la guerra al continente. Los conflictos surgidos en los Balcanes y en el espacio Pos-soviético, han sido nuevas realidades a las que los europeos nos hemos tenido que acostumbrar. Por ello, este trabajo va a tratar de analizar los siguientes puntos entre los que se incluye la vuelta de la guerra a Europa:

1) La importancia de los antiguos miembros de Pacto de Varsovia para la consecución de sus objetivos de seguridad

2) La percepción de la arquitectura de seguridad tras la redistribución de capacidades provocada por la caída del Muro de Berlín

3) La posición de los actores europeos ante los conflictos (des)congelados en Europa: Kosovo, Georgia y Ucrania.

La elección de estos tres asuntos se debe a que, a mi entender, se trata de los puntos que más controversia han generado en lo que a la seguridad europea se refiere. Esencialmente se trata de los principales puntos de fricción entre Rusia y Occidente ya que desde 1989, como prioridad estratégica reconocida en varios documentos oficiales, Moscú ha tratado de frenar la incorporación de los antiguos socios del bloque Oriental a las estructuras de segurida occidentales (OTAN y UE esencialmente). Para ello, Rusia se ha valido de una serie de conflictos (Kosovo, Georgia y Ucrania) congelados que si bien nunca se han resuelto del todo, tampoco han sido guerras prolongadas durante años. Por ello, he considerado que estos tres puntos deberían centrar la atención de este trabajo.

\section{EI Análisis de la Transformación Europea tras la caída del Muro de Berlín}

Aunque el fin de este trabajo es el dar una visión de conjunto de los últimos 30 años en Europa, vamos a dividir este periodo en cuatro etapas diferentes ya que se trata de responder a preguntas tales como cuáles han sido cambios y sobre todo la evolución de la seguridad europea. Por ello, vamos a analizar los tres aspectos anteriormente señalados en cada una de las siguientes fases:

1) Adaptación a la nueva realidad europea (1989-2000)

2) Guerra al Terrorismo (2001-2004)

3) Final de la Luna de Miel (2005-2013)

4) Divorcio y comienzo de la escalada (2014-2019) 


\section{Adaptación a la nueva realidad europea (1989-2001)}

El final de la URSS se gestó cuando se pasó de la Doctrina Breznev a la Doctrina Sinatra ${ }^{58}$ o lo que es lo mismo, cuando la URSS decidió no intervenir allí donde se detectara «una fuerza hostil al socialismo». Si bien es cierto que desde la llegada de Gorbachov se detectaron indicios de este cambio, el punto en el que esta tendencia tácita se hizo expresa fue en su famoso discurso de diciembre de 1988 en la Asamblea General de las Naciones Unidas ${ }^{59}$. En Nueva York, Gorbachov confirmó que nunca más intervendrían en Europa del Este para cambiar el curso de la política ${ }^{60}$. La principal consecuencia de esta decisión fue la caída del Muro de Berlín, un hecho que provocó una absoluta transformación de las relaciones interestatales no solo en Europa, sino en todo el mundo. Sin embargo, debido a que Europa era la zona principal de disputa entre Occidente y la URSS, fue aquí donde se apreciaron de forma más claras las consecuencias de este cambio de tendencia o de paradigma. Analicemos por tanto estas consecuencias de acuerdo con los tres criterios anteriormente destacados:

\subsection{La importancia de los antiguos aliados soviéticos}

Podemos afirmar que los Estados que mostraron un mayor y más pronto interés en incorporarse a las estructuras de seguridad europea - UE y OTAN - fueron aquellos en los que se vivió un mayor grado protesta contra la tutela soviética, es decir, Chequia, Hungría y Polonia. Así, los mandatarios de estos tres estados mostraron no solo su interés en cooperar ${ }^{61}$ con la alianza, sino que declararon su deseo de convertirse en miembros de pleno derecho de la OTAN ${ }^{62}$. Aunque no puede considerarse como un nuevo Estado, en esos primeros momentos la principal preocupación de todas las partes era el futuro de la RDA ya que su integración en la RFA implicaría de forma automática que el territorio de la Alemania Oriental quedaría bajo el paraguas de la OTAN. Este hecho junto con el control de las armas nucleares repartidas por el territorio soviético, centraron la atención de los mandatarios rusos hasta la primera mitad de los años 90 . Concreta-

${ }^{58}$ El término fue acuñado por el portavoz del Ministerio de Asuntos Exteriores de la URSS - Gennadi Guerásimov - en una entrevista en una televisión de los Estados Unidos.

59 Discurso de Gorbachov en la ONU anunciando medidas soviéticas de desarme. Disponible en http://www.historiasiglo20.org/TEXT/gorbachovONU1988.htm Acceso 12 de febrero de 2019.

60 Alan R. Collins, «Gorbachev and the End of the Cold War», Review of International Studies, no. 24 (1998): 201-219.

61 «Walesa asks NATO "Partnership"», Chicago Tribune, 4 de julio de 1991.

62 «zech Leader Pushes for Open NATO» New York Times, 22 octubre de 1993. 
mente, hasta la aparición de Informe de Ampliación de la OTAN de 1995 algo que se tradujo en el primer punto discrepancia grave entre la Federación Rusa y los Estados Unidos. Esta discrepancia fue tan honda que llevó incluso a la suspensión de la cooperación de Moscú con la OTAN en los órganos y programas ${ }^{63}$ creados a tal efecto ${ }^{64}$.

En 1995 la Alianza hizo expreso su interés de incorporar a los territorios de Chequia, Polonia y Hungría al espacio OTAN, algo que no solo no convencía a Rusia, sino que además alertaba a Moscú de la posibilidad de incorporación de otros Estados que habían formado parte de la propia URSS. Es decir, se alertaba sobre la posibilidad de que el Báltico ${ }^{65}$ pudiera llegar a formar parte de la OTAN, ampliando así la Alianza hasta las mismas puertas de Rusia ${ }^{66}$.

Si bien es cierto que Moscú mostró su más enérgica protesta ante lo que era ya la primera ampliación de la OTAN al Este (1997), no es menos cierto que los problemas internos rusos no le permitieron plantear una resistencia mayor. Esencialmente podemos destacar tres grandes problemas que limitaron su capacidad de actuación:

a) La inestabilidad política interna provocada por un Partido Comunista que en 1995 ganó las elecciones legislativas.

b) La guerra abierta en Chechenia (1994-1996) que se cobró casi 100.000 vidas y que minaba la popularidad de Yeltsin.

c) La crisis financiera rusa que provocó la salida definitiva del gobierno ruso de los llamados occidentalistas en favor de los eslavófilos que ya entonces estaban liderados por Vladimir Putin.

No obstante, y a pesar de sus discrepancias, Rusia comenzó a desarrollar un nuevo modelo de relación con OTAN en el que Moscú obtenía beneficios a cambio de aceptar ampliaciones hacia el Este ${ }^{67}$.

\subsection{La percepción de la arquitectura de seguridad}

Las organizaciones que más seriamente se vieron afectadas por la caída del Muro de Berlín fueron el Pacto de Varsovia y la OTAN. La primera porque se vio obligada a disolverse y la segunda porque tuvo que transfor-

${ }^{63}$ Nos estamos refiriendo a la Parnetship for Peace y al EuroAtlantic Partnership Council.

64 James Goldgeier: Not Whether but...

65 «Albright says 3 Baltic states "serious candidates"» Chicago Tribune, 14 julio 1997.

${ }^{66}$ El caso más claro fueron las repúblicas bálticas. Ver Stephen Blank, «Russia...115-125.

${ }^{67}$ Ronald D. Asmus: Opening NATO's Door. How the Alliance Remade Itself for a New Era, (New York: Columbia University Press, 2002=. 
marse para adaptarse a la nueva realidad que nacía en Europa tras el fin de la URSS. Quizás el mayor cambio quedó plasmado con la aprobación del Concepto Estratégico aprobado en Roma de $1991^{68}$ al reconocer éste que no existía ninguna amenaza estatal, lo que ponía fin a la Guerra Fría (ASMUS et al, 1993).

En lógica con esta declaración, la OTAN inició un proceso de transformación institucional por el que adaptaba sus órganos a la nueva realidad cooperativa que se iniciaba en Europa. En un primer momento se creó una versión cooperativa en todos los foros de la Alianza, y posteriormente se crearon programas (Partnership for Peace) y órganos (Euro Atlantic Partnership Council) exclusivos para los socios de la OTAN. En los casos de Rusia y Ucrania, debido a su especial importancia para la seguridad de Europa, la Alianza estableció marcos de cooperación singularizados ${ }^{69}$ que les diferenciaban de los programas ofrecidos al resto de socios. De esta forma la alianza se adaptaba a la nueva realidad europea y trataba así de compensar a Rusia por la ampliación que en 1999 convertía a Chequia, Hungría y Polonia en miembros de pleno derecho de la OTAN. No obstante, la Alianza Atlántica no cesó en su transformación organizativa y funcional ya que debía adaptarse a una realidad internacional con un concepto de seguridad mucho más amplio y donde los enemigos no eran visibles. Para tal efecto y coincidiendo con su 50 aniversario, en 1999 la OTAN aprobaba un nuevo concepto estratégico que le habilitaba a buscar su seguridad más allá del área cubierta por el artículo 5 del Tratado de Washington ${ }^{70}$.

También la UE y la OSCE trataron de adaptarse a la nueva realidad. La UE comenzó a desarrollar su proyección exterior tanto en lo que a la política exterior (Tratado de Maastricht) como a la de seguridad (Tratado de Ámsterdam) se refiere. La UE tenía varios retos que debía asumir como eran la integración de la UEO en su seno, evitar la duplicidad de funciones y capacidades con la OTAN, así como la armonización de las diferentes culturas estratégicas de los diferentes miembros de la UE al nuevo proyecto de acción exterior que se abría con la PESC. Un elemento fundamental para la PESC fue la Cumbre bilateral de Saint Malo donde el Reino Unido y Francia se abrían a construir una política de defensa europea ${ }^{71}$.

68 Disponible en https://www.nato.int/cps/en/natohq/official_texts_23847.htm

${ }^{69}$ En el caso de Rusia se firmó el «NATO-Russia Founding Act» y en el de Ucrania la «Charter on a Distinctive Partnership between Ukraine and NATO»

70 Antonio Marquina (Ed.): La OTAN en su...

71 Tras la Cumbre de Saint Malo (1997) en los Consejos Europeos de Colonia (1999), Helsinki (1999) y Marsella (2001) se pusieron las bases de la futura defensa europea poniendo las bases de la integración de la UEO en la UE, aprobado los Helsinki Headline Goal y la Fuerza de Reacción Rápida. 
La caída del Muro de Berlín permitió que la OSCE pasara de ser un régimen internacional para convertirse en una organización internacional. Este hecho se consumó en 1994 en la Cumbre de Budapest, pero desde 1989 se fueron dando pasos en este camino como fueron la aprobación de la Carta de París (1989), la celebración del primer Consejo de Ministros (1991) o el envío de su primera misión hacia exterior en Kosovo, Sandjak y Voljvodina (1992).

\subsection{Los conflictos en Europa}

Si bien es cierto que la caída del Muro de Berlín supuso una cierta relajación en la percepción de la amenaza, tampoco podemos olvidar que el fin de los bloques trajo de vuelta la guerra a Europa. En los años 90 Europa fue escenario de varios conflictos armados lo que nos recordó que la guerra seguía siendo una herramienta de las relaciones internacionales viva y a disposición de los actores del sistema internacional. El comienzo de los 90 nos trajeron guerras en Moldavia, Georgia, Nagorno-Karabaj ${ }^{72}$ y sobre todo en Yugoslavia un estado que tras la proclamación de independencia de Eslovenia y Croacia se desvanecía como un castillo de naipes. Si bien los tres primeros casos pueden ser considerados como consecuencias de la desintegración soviética, el caso yugoslavo fue un torpedo en la línea de flotación de Europa no solo como civilización, sino también como proyecto político de integración.

a) Como civilización hay que decir que Europa había entrado en la fase de las sociedades post-heroicas donde realidades como la guerra, la limpieza étnica e incluso el genocidio permanecía fuera del imaginario colectivo

b) ...y como proyecto político cuestionaba la viabilidad de una PESC que comenzaba a dar sus primeros pasos. Esta falta de viabilidad quedó reflejada en la tristemente famosa frase pronunciada por el entonces presidente de turno y ministro de exteriores luxemburgués Jacques Poos quien tras los primeros combates en Yugoslavia afirmó que «This is the hour of Europe. It is not the hour of the Americans $^{73} . »$

Europa quedó seriamente tocada tanto en lo moral como en lo político, sobre todo, tras episodios tan horrorosos como fueron Srebrenica o la matanza del Mercado de Sarajevo. Quizás por ello, cuando en 1999 se empezó

72 Dov Lynch: Russian peacekeeping in the CIS. The cases of Moldova, Georgia and Tajikistan, (London: MacMillan Press, 2000).

73 The New York Times, 29 de junio de 1991. 
a conocer la situación de limpieza étnica que estaban sufriendo los kosovares a manos de los serbios, la presión de las sociedades europeas recayó diretamente sobre sus políticos para evitar otra situación similar. Sin embargo, ya nadie pensó que era «la hora de Europa» porque todo el mundo estuvo de acuerdo en que había llegado la hora de la OTAN. Por primera vez la Alianza Atlántica llevaba a cabo una operación militar que además estaba fuera del área del artículo 5. Se ponía así en funcionamiento el nuevo Concepto Estratégico aprobado en Washington solo unos días antes ${ }^{74}$.

La incorporación de Hungría, Polonia y Chequia a la OTAN — sobre todo la intervención en Kosovo - supuso el inicio de una nueva etapa en las relaciones Occidente-Rusia marcada por la tensión y la enemistad. Sin embargo, esta tensión se vio interrumpida por la emergencia de una amenaza inesperada: el terrorismo islámico internacional. Este hecho permitió un paréntesis antes de la ruptura definitiva entre Rusia y Occidente, marcando una breve etapa de distensión entre las partes. No obstante, hay que señalar que justo al final de esta etapa, y ya con Putin como presidente, se dieron algunos pasos hacia la confrontación como la restauración del himno soviético o la aprobación de una Nueva Doctrina Militar ${ }^{75}$ (2000) que apuntaba al nuevo y agrsivo posicionamiento de Rusia ${ }^{76}$ en Europa.

\section{Guerra al Terrorismo (2001-2004)}

El 11 de septiembre de 2001 a las 8:46 un Boeing 767 que había despegado del Aeropuerto Internacional de Logan se estrellaba contra la Torre Norte del complejo WTC y solo 16 minutos después, otro avión hacía lo mismo en la Torre Sur. A las 9:39 un tercer avión impactaba contra el Pentágono y 24 minutos más tardes un cuarto avión caía en campo abierto en Shanksville (Pensilvania) El terrorismo islámico internacional emergía en medio de la confrontación entre Rusia y Occidente ${ }^{77}$ provocando un paréntesis de tres años.

Si bien los atentados de Nueva York y Washington fueron los más espectaculares, lamentablemente no fueron los únicos ni mucho menos los úl-

74 «The Alliance's Strategic Concept» 24-4-1999. Disponible en https://www.nato.int/ cps/ie/natohq/official_texts_27433.htm

${ }^{75}$ La Doctrina Militar del 2000 que es una revisión de la de 1997 puede consultarse en su versión en inglés en https://www.armscontrol.org/act/2000_05/dc3ma00

76 José Miguel Palacios, y Paloma Arana, «Doctrina militar rusa: herencia soviética, realidades postsoviéticas, perspectiva europea», Revista CIDOB d'Afers Internationals, no. 59 (2002): 81-103, p. 82 .

77 Kara Bosworth, «The Effect of 11 September on Russia-NATO Relations», Perspectives on European Politics \& Society, no. 3 (2002): 361-387. 
timos. La Federación Rusa también sufrió la lacra del terrorismo con un total de 10 atentados (2002-2004) que dejaron un saldo de 1024 muertos con atentados tan crueles como el ocurrido en Teatro Dubrovka o en la Escuela número de 1 de Beslán.

\subsection{La importancia de los antiguos aliados soviéticos}

En este contexto de lucha contra el terrorismo, cabe destacar un breve periodo de distensión que a la OTAN permitió llevar a cabo lo que se ha denominado La Gran Ampliación. En noviembre de 2002 la Alianza Atlántica, con el beneplácito de una Rusia que a duras penas se mantenía en pie, invitaba a convertirse en miembros a siete estados que bien habían pertenecido directamente a la URSS (Estonia, Letonia y Lituania) o que habían estado en la órbita de la URSS (Eslovaquia, Eslovenia, Bulgaria, Rumanía) A cambio de «permitir» esta ampliación, Rusia podía empleaser con dureza contra el terrorismo checheno. Además, Moscú recibió una compensación en forma de participación privilegiada en la Alianza: el Consejo OTAN-Rusia. Así, se creó un órgano que permitía a Moscú sentarse en la misma mesa que los 19 miembros de la OTAN, aunque no se le permitía votar las decisiones de una Alianza Atlántica que parecía transformarse para adaptarse a la amenaza terrorista.

En el año 2004 - coincidiendo con la Gran Ampliación-, Estonia, Letonia, Lituania, Polonia, Chequia, Hungría, Eslovaquia y Eslovenia también se incorporaron a la UE como miembros de pleno derecho. Aunque desde el punto de vista político y económico esta ampliación supuso una revolución, desde el punto de vista de la defensa no implicó un cambio tan significativo ya que la defensa europea todavía no estaba plenamente construida y por ello a corto plano sus consecuencias fueron mucho menores.

\subsection{La percepción de la arquitectura de seguridad}

El 11 de septiembre también transformó la percepción de la seguridad en el espacio Euro-Atlántico. De hecho, la primera vez que se invocaba el art. 5 del Tratado de Washington se hacía para hacer frente no a un estado sino a una amenaza no tradicional como es el terrorismo. Por ello, un año después de 11s, en la Cumbre de Praga (Noviembre 2002) la OTAN inició un proceso de transformación que le llevó revolucionar su propia estructura de mandos $^{78}$, a crear una fuerza de reacción rápida (NATO Response Force)

78 LA OTAN «desterritorializó» su estructura de mando deshaciendo su tradicional entre el mando europeo (SACEUR) y el americano (SACLANT). La OTAN pasaba a tener un mando operativo (ACO) y otro doctrinal para llevar a cabo la transformación (ACT) 
o a adaptar su doctrina a esta nueva amenaza invisible (Policy Guidelines on Counter-Terrorism ${ }^{79}$ ).

Por su parte, la UE también se fue adaptando a esta nueva situación, aunque le resultó más fácil ya que su política exterior y de seguridad apenas había comenzado a fraguarse. En este sentido en el año 2003, la UE aprobaba la Estrategia Europea de Seguridad - más conocido por «el Documento Solana»- que llevaba por subtítulo una Europa Segura en un Mundo Mejor ${ }^{80}$. Tanto este documento como el Concepto Estratégico de la OTAN aprobado en Washington en 1999 no solo afirmaban que las amenazas no eran las tradicionales, sino que descartaban una confrontación con Rusia.

\subsection{Los conflictos en Europa}

En esta segunda etapa los conflictos en Europa entraban en la «fase de post-conflicto» que sirvió para que la OTAN cediera el testiga a la UE en lo que las misiones de paz se refiere. Esta transición en el mando ocurrió tanto en Bosnia, donde la SFOR dejó paso a la EUFOR-Althea (2004) como en Macedonia, donde la Allied Harmony también fue sustituida por la $E U$ FOR-Concordia (2003).

Si bien en lo que a los conflictos en Europa se refiere este segundo periodo fue tranquilo, aunque lejos de nuestras fronteras se gestó una intervención internacional que minó no solo la relación de Occidente con Rusia sino los propios consensos de la defensa europea. Lógicamente me estoy refiriendo a la intervención norteamericana en Irak (2003).

\section{Final de la Luna de Miel (2005-2014)}

A partir de 2004 la actitud de Moscú fue pasando gradualmente de la cooperación al conflicto, volviendo así a la dinámica adoptada a finales de los años 90. Aunque el cambio no se hizo público hasta el famoso discurso de Vladimir Putin en la Conferencia de Seguridad de Munich ${ }^{81}$ (2007), algunos años antes ya se atisbaban claras señales de hostilidad de Rusia hacía Occidente $^{82}$, hostilidad que estaba esencialmente provocada por la sensación de cerco derivada de las ampliaciones de la OTAN hacia el Este.

\footnotetext{
79 Disponible en https://www.nato.int/cps/en/natolive/topics_69482.htm

80 Disponible en https://www .consilium.europa.eu/media/30808/qc7809568esc.pdf

81 Disponible en http://en.kremlin.ru/events/president/transcripts/24034

82 Edward Lukas: The New Cold...
} 


\subsection{La importancia de los antiguos aliados soviéticos}

Si bien durante esta tercera fase las ampliaciones culminadas (Croacia y Albania en 2009) no fueron especialmente controvertidas, las frustradas (Georgia, Ucrania y Macedonia) marcaron un antes y un después en las relaciones entre Bruselas y Moscú. Dejando a un lado a Macedonia cuya dificultad estribaba en su polémico nombre ${ }^{83}$, los casos de Georgia y Ucrania por su importancia estratégica suscitaron los peores temores en Moscú, ya que ambos países son percibidos como fundamentales para la seguridad rusa.

Tras las revoluciones de la Rosa (2003) y Naranja (2004) Georgia y Ucrania cambiaron su alineación en política exterior convirtiéndose en aliados incondicionales tanto de los Estados Unidos como de la OTAN. Una vez se produjo los cambios de gobierno en Ucrania en 2006 y Georgia en 2004, sus embajadores declararon de forma oficial su intención de integrarse en la OTAN. El país caucásico, que llevaba mucho tiempo trabajando en la interoperabilidad a través del programa Partnership for Peace, esperaba ser invitado a la integración en la cumbre de Bucarest de 2008. Sin embargo, la invitación no solo no se produjo, sino que en agosto de ese año Georgia tuvo que afrontar el ataque de Rusia alejando así sus aspiraciones de convertirse en miembros de la OTAN. Los que en cambio sí que pudieron iniciar su proceso de integración en Bucarest fueron Croacia y Albania proceso culminarían el proceso en 2009.

\subsection{La percepción de la arquitectura de seguridad}

La cooperación de la OTAN con Georgia y Ucrania cambió la percepción de seguridad de Rusia. Así, tras la declaración de intenciones de Georgia de convertirse en miembro de la OTAN, Rusia tomó una serie de medidas entre las que podemos destacar el bloqueo económico, la deportación de ciudadanos georgianos residentes en Rusia y la más importante: la retirada de Rusia del Tratado de Fuerzas Convencionales en Europa.

Además, desde 2008 Rusia comenzó a construir un sistema de denegación de acceso, un sistema $\mathrm{A} 2 / \mathrm{AD}^{84}$ que tiene por principal objetivo limitar los efectos que puedan tener las ampliaciones de la OTAN sobre su seguri-

${ }^{83}$ Utilizaremos el nombre de Macedonia aunque en el periodo tratado en este trabajo el internacionalmente aceptado era el del Antigua República Yugoslava de Macedonia (FYROM)

${ }^{84}$ Stephan Frühling and Guillaume Lasconjarias, «NATO A2/AD and the Kaliningrad Challenge», no. 2 (2016): 95-116 esp. p. 96. 
dad. Esta iniciativa está basada en el establecimiento de sistema antiaéreos en cinco puntos geográficos concretos:
a) La Península de Kola
b) El enclave de Kaliningrado
c) Osetia y Abjasia
d) La península de Crimea
e) La base de Tartús (Siria)

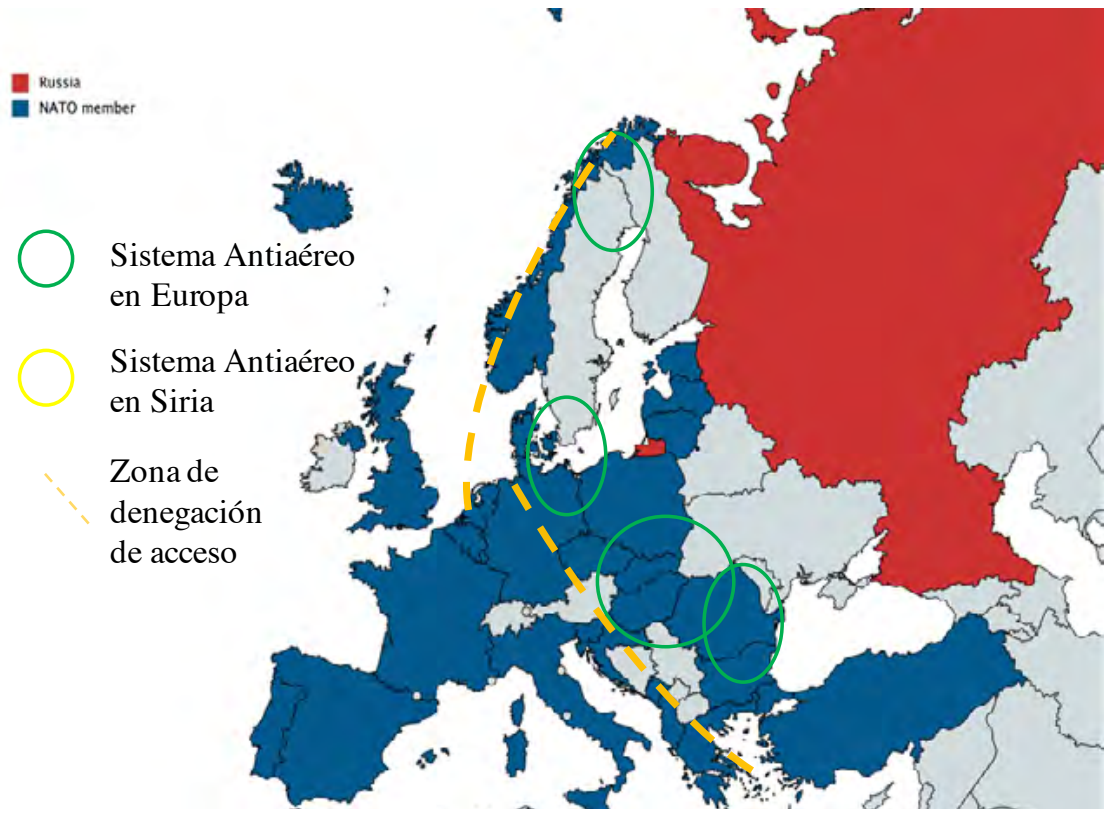

Gráfico 1

La construcción de la zona A2/AD

Fuente: Elaboración Propia

La construcción de la zona A2/AD trata de evitar potenciales ataques aéreos - o con misiles - contra Rusia desde territorio OTAN ${ }^{85}$. La medida está basada en la percepción de inseguridad que ve el entorno de $\mathrm{Pu}$ -

85 Fabrice Pothier, «An Area-Access Strategy for NATO», Survival, no. 3 (2017): 73-80 esp. p.86. 
tin tras los cambios políticos acaecidos en Serbia, Irak y Libia. Concretamente, con esta medida el Kremlin pretende evitar que una campaña aérea liderada por la OTAN pueda servir de apoyo a una insurrección interna que tenga por meta el derrocamiento del gobierno tal y como ocurrió en los casos anteriormente mencionados ${ }^{86}$. Si bien es cierto que la construcción de esta zona de denegación de acceso ha estado motivada por las imparables ampliaciones de la OTAN y sus posibles repercusiones en cambios de regímenes, también hay que señalar que la zona A2/AD es una protección o una reacción al escudo antimisiles que la Alianza comenzó a construir en 2010.

En todo caso, lo que queda claro es que al final de esta etapa las relaciones entre ambos bloques quedaron prácticamente rotas tal y como posteriormente probaron hechos como la aprobación de la Nueva Doctrina Mi$\operatorname{litar}^{87}$ rusa (2010), los ejercicios ZAPAD ${ }^{88}$ (2013), la intervención de la OTAN en Libia (2011) o el caso Snowden.

En lo que a la Unión Europea se refiere, Bruselas siguió trabajando en la construcción de la defensa europea manteniendo su propio ritmo y sobre todo apostando por medidas firmes y consensuadas como la creación de los Battle Groups, la extinción definitiva de la UEO o la entrada en vigor del Tratado de Lisboa lo que dotaba a Katherine Ashton poderes casi plenos en política exterior y en defensa de la UE.

La OSCE fue la organización que más sufrió el recrudecimiento del conflicto entre Rusia y Occidente. Si los años 90 fueron una década en la que la organización creció mucho, en la primera década del siglo XXI la OSCE quedó paralizada. De hecho en este periodo solo se ha celebrado una cumbre, la de Astaná, que además tuvo unos resultados muy modestos.

\subsection{Los conflictos en Europa}

Durante esta etapa que hemos denominado «el final de la luna de miel», los conflictos que marcaron la seguridad europea fueron, sin lugar a duda, los de Georgia y Ucrania. En ambos casos, la decisión se tomó después de que Rusia asistiera como testigo a las Revoluciones de Colores. En todo caso lo que se pretendía era frenar la influencia occidental en el espacio post-soviético, lo que a su vez servía para construir una nueva arquitectura de seguridad que bien podríamos llamar de Post-Guerra Fría. Lo que en un primer momento parecía un intento de recuperación del honor per-

${ }^{86}$ Samuel Charap y Jeremy Sapiro, «Consecuences of a New Cold War», Survival, no. 2 (2015): 37-46, esp. p. 39.

87 En la nueva Doctrina Militar se planteaba que la OTAN era una amenaza.

${ }^{88}$ En los ejercicios militares ZAPAD de 2013 se simulaba una invasión rusa de Polonia. 
dido en 1991 a través de la recuperación de territorios históricos del denominado «Extranjero Próximo», en realidad formaba parte de una estrategia más elaborada que no solo alejaba a Georgia y a Ucrania de la OTAN, sino que los convertía en parte de un complejo sistema de defensas antiaéreas que busca reducir eficacia y valor a las ampliaciones llevadas a cabo por la Alianza Atlántica desde 1999. Para lograr ese fin, Moscú se vio obligado a ocupar tres territorios - Abjasia y Osetia del Sur y Crimea - donde posteriormente se desplegarán sistemas antiaéreos que forman parte del A2/AD.

Si bien es cierto que las acciones de Rusia no encontraron justificación ni en el seno de la OTAN, ni en el de la UE, sí que lograron provocar división en lo que a la respuesta se refiere. Si bien en la OTAN las consecuencias se redujeron a que el grupo de los nuevos miembros reclamó medidas de protección para evitar sufrir situaciones similares, en la UE las consecuencias fueron mayores. Por un lado, se alinearon el Reino Unido, los países escandinavos y los PECOs con posiciones mucho más contundentes frente a Rusia mientras que por el otro, se alinearon aquellos que a pesar de ser conscientes de la amenaza que supone Rusia prefirieron dar una respuesta más moderada para evitar las consecuencias económicas que podría conllevar una confrontación con Rusia.

Por último, los hechos ocurridos en Georgia y Ucrania certificaron la muerte de la OSCE ya que en los últimos años esta organización había servido como «gestora» de los conflictos congelados. Además la Cumbre de 1999 celebrada en Estambul había sido el marco para la firma de un Memorando entre Rusia y Ucrania en el que se reconocía entre otras cosas que Crimea estaba bajo soberanía de Kiev y que la integridad territorial de Ucrania era inviolable.

Tal y como dijimos al comienzo de este apartado, el punto de inicio de esta etapa estuvo marcada por el discurso pronunciado por Vladimir $\mathrm{Pu}$ tin en Munich (2007). Siguiendo esta lógica podríamos finalizar este apartado con nuevos y agresivos discursos como los pronunciados por Putin en el club Valdai ${ }^{89}$ en Sochi (24-20-2014), ante la Duma Estatal ${ }^{90}$ (18-3-2014) o en la tribuna del Kremlin durante la celebración del Día de la Victoria ${ }^{11}$ (8-5-2014). Todos estos hechos apuntan a que al menos para el Kremlin la Guerra Fría parece no haber acabado ${ }^{92}$.

89 «Discurso ante el Club Valdai en Sochi». Disponible en http://en.kremlin.ru/events/ president/news/58848

90 «Discurso ante la Duma Estatal». Disponible en http://en.kremlin.ru/events/president/ news $/ 20603$

91 «iscurso por el Día de la Victoria». Disponible en http://en.kremlin.ru/events/president/news/20989

92 Robert Legvold, The Return to the Cold War, (London: Polity, 2016). 


\section{Divorcio y comienzo de la escalada (2014-2019)}

Desde la invasión de Ucrania en febrero de 2014 hasta septiembre de 2015, vivimos un periodo de unos 20 meses en el que se alcanzaron las máximas cotas tensión entre Occidente y Rusia. En este periodo se identificaron hasta 77 incidentes de los cuales 11 fueron calificados como Serious Incident $t^{93}$ y por lo tanto susceptibles de provocar una escalada internacional (FREAR, 2015). Aunque estos incidentes nunca llegaron a mayores, al menos tres hechos sí que tuvieron consecuencias graves, aunque afortunadamente no provocaron una escalada. En concreto me estoy refiriendo al derribo del avión de pasajeros de Malaysian Airlines MH17 (junio 2014), a la interceptación de un cazo ruso que había violado el espacio aéreo turco (noviembre 2015) y al secuestro de un oficial de inteligencia estonio (septiembre 2015)

\subsection{La importancia de los antiguos aliados soviéticos}

Todos estos hechos anteriormente descritos hicieron cambiar el rol que los países de Europa Central y Oriental estaban desempeñando hasta la fecha en la OTAN. Si bien en un primer momento estos nuevos miembros supusieron un activo para la Alianza en lo que a la relación con Rusia se refiere, en los últimos años las políticas debido a las agresivas del Kremlin, estos estados han transformado su rol de éstos dentro de la Alianza pasando de ser proveedores a demandantes netos seguridad. Esta nueva situación es uno de los factores que han provocado que la Alianza Atlántica haya cerrado su política de ampliación tal y como demuestra que en los últimos años tan solo Montenegro haya podido culminar su integración en la OTAN. No obstante, la política de freno de las ampliaciones no ha venido acompañada de una reducción de la hostilidad por parte de Rusia. De hecho, Rusia intentó boicotear la integración de Montenegro apoyando el intento de asesinato del primer ministro Djukanovic ${ }^{94}$.

${ }^{93}$ Entre estos 11 Serious Incident podemos destacar dos tanto por su especial gravedad como por el lugar donde se desarrollaron, Suecia un país neutral que no está involucrado en las actividades de la OTAN. El primero se desarrolló en la bahía de Estocolmo (18-10-2014) cuando apareció un submarino nuclear ruso en las costas suecas (The Guardian, 19-10-2014, disponible en https:// www.theguardian.com/world/2014/oct/19/sweden-search-russian-submarine-stockholm).

El segundo incidente ocurrió en el Aeropuerto Internacional de Malmoe (12-12-2014) cuando un caza ruso entró en el espacio aéreo del aeropuerto sin llevar activado el transponder, por lo que estuvo a punto de provocar un accidente con un avión de pasajeros que se vio obligado a realizar un aterrizaje de emergencia. (The Local 13-12-2014, disponible en https:// www.thelocal.se/20141213/near-miss-in-swedish-airspace-concerns-military)

${ }^{4}$ Ver The Telegraph, 18-2-2017, disponible https://www.telegraph.co.uk/news/2017/02/18/ russias-deadly-plot-overthrow-montenegros-government-assassinating/ 
Aunque no pueden ser considerados como candidatos oficiales a la Alianza, Finlandia y Suecia comienzan a plantearse la opción de abandonar su neutralidad e integrarse en la OTAN como forma de protegerse frente a una Rusia cada vez más agresiva.

\subsection{La percepción de la arquitectura de seguridad}

Como consecuencia de esa amenaza detectada en el flanco este, la OTAN se vio obligada a transformar sus políticas de seguridad con el fin de poder dotar de protección a aquellos miembros que se sienten amenazados por Rusia. Para ello, entre las Cumbres de Newport ${ }^{95}$ (2014) y Varsovia ${ }^{96}$ (2016) se aprobaron dos iniciativas, que conforman un paquete denominado Readiness Action Plan, que trata convertir a la OTAN en una organización más disponible y activa frente a la amenaza rusa ${ }^{97}$ :

a) La Very High Readiness Joint Task Force (VJTF) o Spearhead Force busca reducir los tiempos de respuesta de la Nato Response Force (NRF) con el fin de repeler una posible invasión como las ocurridas en Georgia o en Ucrania.

b) La Enhanced Forward Presence (EFP) es una iniciativa para desplegar de forma permanente (en el Báltico, en Polonia, en Rumania y Bulgaria) fuerzas OTAN para evitar la promoción de insurrecciones promovidas por potencias extranjeras utilizando para ello a las diásporas que viven en territorio OTAN y por el otro, disuadir a Rusia de anexionarse los países fronterizos.

Junto a estas dos iniciativas durante este periodo también se completó el despliegue de capacidades defensivas en la ciudad rumana de Deveselu (2016) y en la polaca de Redzikowo (2018) que culminaban la última fase del proyecto del Escudo Antimisiles de la OTAN.

Una de las muestras más claras de la existencia de esta tensión son los masivos ejercicios militares llevados a cabo en los últimos años tanto por la OTAN como por Rusia. En concreto hay que destacar los Trident-2015 llevados a cabo por la Alianza que movilizaron a un total de 45.000 soldados. Por otro lado, Rusia movilizó a 100.000 efectivos en el marco de los ejercicios ZAPAD-2018 en los que además también participaron fuerzas extranjeras.

95 «Wales Summit Declaration», 4-9-2014. Disponible en https://www.nato.int/cps/en/ natohq/official_texts_112964.htm

96 «Warsaw Summit Communiqué» 9-7-2016. Disponible en https://www.nato.int/cps/ en/natohq/official_texts_133169.htm

97 Jens Ringsmose y Sten Ryning: «Now for the Hard Part: NATO's Strategic Adaptation to Russia», Survival, no. 3 (2017):129-146, esp. p. 130. 
En lo que a la Unión Europea se refiere, si bien es cierto que se mantiene la preocupación por la posición de Rusia, a día de hoy, la UE tiene que hacer frente a un desafío más inmediato: el Brexit. En todo caso Bruselas está trabajando en una serie de acciones que concretan y adaptan el proyecto de defensa común a un nuevo entorno estratégico. En concreto, me gustaría destacar tres iniciativas que, en mi opinión, marcará el futuro de la seguridad y defensa en Europa:

a) La Estrategia Global para la Política Exterior y de Seguridad de la $\mathrm{UE}^{98}$ más conocida como el Documento Mogherini

b) La puesta en marcha de la Cooperación Reforzada (PESCO)

c) La declaración conjunta OTAN/UE sobre la amenaza común.

\subsection{Los conflictos en Europa}

En esta última fase los conflictos de Georgia y Ucrania han marcado las agendas de las organizaciones internacionales de seguridad en Europa tal y como demuestran las continuas alusiones en las declaraciones finales de las diferentes cumbres celebradas durante este periodo. En el caso de la OTAN tanto en la Cumbre de Gales como en la de Varsovia, la Alianza no solo ha sido critica con la anexión de Crimea, sino que ha apostado por el fortalecimiento de la cooperación y del diálogo con los candidatos Georgia y Ucrania. En lo que a la UE se refiere en todas y cada una de las Cumbres UEUcrania celebradas desde 2015, se han incluido duras condenas a la anexión rusa de tierras ucranianas. Por lo tanto, este asunto ha marcado la agenda y el devenir de las dos organizaciones.

En lo que a la OSCE se refiere, en junio de 2014 se produjeron encuentros entre las partes para lograr un alto el fuego en las zonas de Donetsk y Lugansk. Como consecuencia de estos encuentros se firmó el conocido como Protocolo de Minsk ${ }^{99}$ que estaba compuesto por 12 puntos entre los que se incluían además del mencionado alto el fuego, medias tales como intercambios de prisioneros, verificación fronteriza supervisada por la OSCE o acciones de Desmovilización Desarme y Reintegración (DDR) de combatientes ${ }^{100}$. Debido a las continuas violaciones del alto el fuego y a la celebración de elecciones no autorizadas en Donetsk y Lugansk, el Protocolo de Minsk no ha tenido el efecto pretendido y de nuevo la credibilidad de la organización se ha puesto en cuestión.

${ }^{98}$ Disponible en https://europa.eu/globalstrategy/sites/globalstrategy/files/eugs_es_version.pdf

99 Disponible en https://www.ft.com/content/21b8f98e-b2a5-11e4-b234-00144feab7de

100 Otto Muller, The Ukraine Crisis and Russia's Attitude towards the Organization for Security and Co-operation in Europa, GRIN Verlag, 2017. 
En todo caso debemos decir que los conflictos que Georgia y Ucrania mantienen con Rusia no solo han alterado la percepción de seguridad en Europa, sino que están marcando las agendas de la OTAN y de la UE.

\section{Conclusiones}

Cuando se cumplen 30 años de la caída del Muro de Berlín Europa debe reflexionar sobre cuáles han sido los cambios en materia de seguridad como ésta ha evolucionado. La primera conclusión que podemos extraer es que Europa se enfrenta a un escenario completamente distinto. Quizás el hecho más chocante para nuestras sociedades post-heroicas y post-modernas haya sido volver a convivir con el cruel fenómeno de la Guerra (Yugoslavia, Nagorno-Karabakh, Moldavia, Georgia y finalmente Ucrania) algo que había desaparecido de nuestras vidas y cuyas consecuencias han agitado los cimientos de la pacífica Europa.

Por otro lado, cuando creíamos haber derribado el «Telón de Acero» nos hemos dado cuenta de que esta construcción artificial solo se había desplazado unos miles de kilómetros hacia el este, concretamente a la frontera con Rusia ya que con la incorporación de 14 nuevos estados la OTAN le ha ganado a Rusia 1.286.418 kilómetros cuadrados ${ }^{101}$ pero no ha logrado acabar con el conflicto. Esta nueva realidad ha estado en la base de la reconstrucción de la política de seguridad rusa que tiene por fin la recuperación de rol hegemónico no solo en Europa sino también en el mundo. Para ello, Moscú ha elaborado nuevas doctrinas militares, ha ocupado ilegalmente territorios, ha modernizado a sus fuerzas armadas y ha abandonado algunos de los principales tratados de desarme como el CFE o el INF. Todas estas acciones parecen desembocar en la construcción de una zona de denegación de acceso cuyo objetico no es otro que minimizar el efecto que han tenido las ampliaciones en la seguridad nacional rusa.

Por su parte, la OTAN ha tratado de adaptarse a una nueva seguridad europea creando nuevos mecanismos para hacer frente a las amenazas no tradicionales sin olvidar su razón de ser: proteger el espacio OTAN de una posible invasión procedente de Rusia. De hecho, la incorporación de los países de Europa Central y Oriental fue concebida como un glacis de seguridad de Europa Occidental, y en los últimos años la agresividad de Rusia ha hecho que los nuevos miembros se conviertan en los principales consumidores de seguridad teniendo incluso que desplegar fuerzas de la Alianza en sus fronteras.

101 Con la única excepción de los ex-yugoslavos y de Albania que se retiró del Pacto de Varsovia el resto de nuevos miembros pertenecieron al Pacto de Varsovia. 
En lo que a la UE se refiere, ésta ha tratado de ir construyendo una política de seguridad propia e independiente pero los diferentes intereses económicos, políticos y de seguridad - de los estados miembros han impedido que se pudiera llevar a cabo en las condiciones que necesitaba Europa. Sin embargo, la UE ha logrado algunos éxitos notables que en el año 1989 hubieran parecido imposible cuando buena parte de sus miembros permanecían bajo el control político y económico de Moscú.

Quizás el actor que menos ha evolucionado en estos 30 años ha sido Rusia ya que, si bien ha cambiado los métodos, los fines siguen siendo los mismos. Lo que antes se denominaba Doctrina Breznev hoy ha pasado a llamarse Doctrina Primakov, pero la realidad es la misma: una soberanía limitada. De hecho, Rusia ya no solo trata de controlar los gobiernos de la entonces llamada Europa del Este sino que interviene en la política de Europa Occidental como prueban los casos del Brexit, Cataluña o el apoyo de Moscú a partidos populista y antisistema.

En este sentido podemos afirmar que si bien Occidente ha adaptado sus capacidades, y sobre todo sus doctrinas, a la nueva seguridad en Europa, Rusia parece seguir anclada en los postulados de la más dura Guerra Fría. Así, la realidad de la seguridad en Europa ha pasado de vivir en un equilibrio (imperfecto) de poder entre bloques a una situación en la que Rusia, ante su falta de capacidad promueve la inestabilidad en aquellos lugares -Georgia, el Báltico, Crimea etc...- en los que Occidente manifiesta interés. De este modo, podemos responder a nuestras preguntas de investigación diciendo que Europa es un continente que si bien ha extendido su comunidad de seguridad, las amenazas exteriores no solo son más peligrosas sino que están más activas que antes de la caída del Muro de Berlín.

\section{Sobre el autor}

Alberto Priego Doctor en Relaciones Internacionales, Licenciado en Ciencias Política y de la Administración - Especialidad en Relaciones Internacionales, con Mención en Estudios Europeos (UCM) . Ha sido visiting scholar en think tanks como East West y universidades extranjeras como, University of Reading, Coimbra, Hebrea de Jerusalem o en la School of Oriental and African Studies donde fue Postdoctoral Felllow. Se incorporó a la Universidad Pontificia Comillas en 2010 donde es profesor agregado en el Departamento de Relaciones Internacionales. Posee un sexenio de investigación (2007-2014) Colabora con asiduidad con diferentes medios de comunicación (El Mundo, El Confidencial, El País, RNE, RTVE, La Sexta, Cope etc...) 


\begin{abstract}
About the author
Alberto Priego holds a PhD in International Relations, Bachelor in Political Science and Administration - Specialization in International Relations, with a mention in European Studies (UCM). He has been visiting scholar in think tanks like East West and foreign universities like, University of Reading, Coimbra, Hebrew of Jerusalem or in the School of Oriental and African Studies where he was Postdoctoral Felllow. He joined the Universidad Pontificia Comillas in 2010 where currently he is an Associate Professor in the Department of International Relations. He has a recognised research period (2007-2014) and collaborates regularly with different media (El Mundo, El Confidencial, El País, RNE, RTVE, La Sexta, Cope etc...)
\end{abstract}




\section{Derechos de autor}

Los derechos de autor (para la distribución, comunicación pública, reproducción e inclusión en bases de datos de indexación y repositorios institucionales) de esta publicación (Cuadernos Europeos de Deusto, CED) pertenecen a la editorial Universidad de Deusto. El acceso al contenido digital de cualquier número de Cuadernos Europeos de Deusto es gratuito inmediatamente después de su publicación. Los trabajos podrán leerse, descargarse, copiar y difundir en cualquier medio sin fines comerciales y según lo previsto por la ley; sin la previa autorización de la Editorial (Universidad de Deusto) o el autor. Así mismo, los trabajos editados en CED pueden ser publicados con posterioridad en otros medios o revistas, siempre que el autor indique con claridad y en la primera nota a pie de página que el trabajo se publicó por primera vez en CED, con indicación del número, año, páginas y DOI (si procede). Cualquier otro uso de su contenido en cualquier medio o formato, ahora conocido o desarrollado en el futuro, requiere el permiso previo por escrito del titular de los derechos de autor.

\section{Copyright}

Copyright (for distribution, public communication, reproduction and inclusion in indexation databases and institutional repositories) of this publication (Cuadernos Europeos de Deusto, CED) belongs to the publisher University of Deusto. Access to the digital content of any Issue of Cuadernos Europeos de Deusto is free upon its publication. The content can be read, downloaded, copied, and distributed freely in any medium only for non-commercial purposes and in accordance with any applicable copyright legislation, without prior permission from the copyright holder (University of Deusto) or the author. Thus, the content of CED can be subsequently published in other media or journals, as long as the author clearly indicates in the first footnote that the work was published in CED for the first time, indicating the Issue number, year, pages, and DOI (if applicable). Any other use of its content in any medium or format, now known or developed in the future, requires prior written permission of the copyright holder. 